\title{
The AMIGA underground muon detector of the Pierre Auger Observatory - performance and event reconstruction
}

\author{
Ana Martina Botti*a,b for the Pierre Auger Collaboration ${ }^{c \dagger}$ \\ ${ }^{a}$ Instituto de Tecnologías en Detección y Astropartículas, CNEA-CONICET-UNSAM, Buenos \\ Aires, Argentina \\ ${ }^{b}$ Karlsruhe Institute of Technology, Karlsruhe, Germany \\ ${ }^{c}$ Observatorio Pierre Auger, Av. San Martín Norte 304, 5613 Malargüe, Argentina \\ E-mail: auger_spokespersons@fnal.gov \\ Full author list: http://www.auger.org/archive/authors_icrc_2019.html
}

\begin{abstract}
The Auger Muons and Infill for the Ground Array (AMIGA) aims to both lower the detection threshold of the Pierre Auger Observatory down to energies of $\sim 10^{16.5} \mathrm{eV}$ and to directly measure the muon content of extensive air showers. AMIGA consists of an array of coupled waterCherenkov and buried scintillation detectors deployed in two superimposed triangular grids of $433 \mathrm{~m}$ and $750 \mathrm{~m}$ spacings. Each underground detector has a total area of $30 \mathrm{~m}^{2}$ buried at a depth of $2.3 \mathrm{~m}$, to shield it from the shower electromagnetic component. The scintillation plane is segmented in plastic-scintillator strips with embedded wavelength-shifter optical fibers coupled to a common optical sensor. Before proceeding to the construction of the full-size array, an engineering array was operated until November 2017 to validate and optimize the design, and to evaluate the performance of the detection system. During this phase, scintillation areas of $5 \mathrm{~m}^{2}$ and $10 \mathrm{~m}^{2}$ and two optical sensors, photomultiplier tubes and silicon photomultipliers, were tested. In this work, we present the status and performance of the array currently equipped with silicon photomultipliers, along with the timing performance and geometry reconstruction of modules equipped with photomultiplier tubes. Analyses and results are based on both laboratory and field measurements. Scintillation areas of $10 \mathrm{~m}^{2}$ and silicon photomultipliers as readout have been selected as the baseline design for the full-scale AMIGA array.
\end{abstract}

36th International Cosmic Ray Conference - ICRC2019

24 July - 1 August, 2019

Madison, Wisconsin, USA

\footnotetext{
* Speaker.

${ }^{\dagger}$ for collaboration list see PoS(ICRC2019)1177
} 


\section{Introduction}

Cosmic rays with energies above $10^{17.5} \mathrm{eV}$ are studied at the Pierre Auger Observatory [1] through a hybrid-detection technique, composed of a fluorescence detector (FD) and a surface detector (SD). The FD has 27 fluorescence telescopes distributed at four sites at the edge of the SD. The SD consists of an array of 1660 water-Cherenkov detectors (WCDs) with a $1500 \mathrm{~m}$ spacing (SD-1500), covering a total area of $3000 \mathrm{~km}^{2}$ and a denser array with a $750 \mathrm{~m}$ spacing (SD-750) extending over $23.5 \mathrm{~km}^{2}$. This smaller array is one of the two components of the Auger Muons and Infill for the Ground Array (AMIGA) enhancement [2]. More recently, an even denser array at a $433 \mathrm{~m}$ spacing (SD-433) with an area of $1.9 \mathrm{~km}^{2}$ is being deployed to extend the SD energy threshold down to $10^{16.5} \mathrm{eV}$.

AMIGA also provides direct measurements of the muon components of the extensive air showers with an underground muon detector (UMD) buried at $2.3 \mathrm{~m}$ associated with each AMIGA SD-750 WCD. Each muon detector consists of three $10 \mathrm{~m}^{2}$ modules segmented into 64 plastic-scintillator strips with wavelength-shifter optical fibers that conduct the light to a photo-detector located at the center of the module [2]. During the prototype phase (finalized in November 2017), an engineering array was deployed for proof of concept, physics validation, and optimization of the detector design. In Fig. 1 the layout of the muon detector engineering array is shown. During this prototype phase, $5 \mathrm{~m}^{2}$ and $10 \mathrm{~m}^{2}$ modules were tested, as well as two different photo-detectors: a 64-pixel multi-anode photomultiplier tube (PMT) and an array of 64 silicon photomultipliers (SiPMs). Upon completion of these tests, $10 \mathrm{~m}^{2}$ modules equipped with SiPMs were chosen for the, currently ongoing, production phase of the UMD, which is also part of AugerPrime, the upgrade of the Pierre Auger Observatory [3]. As a main part of
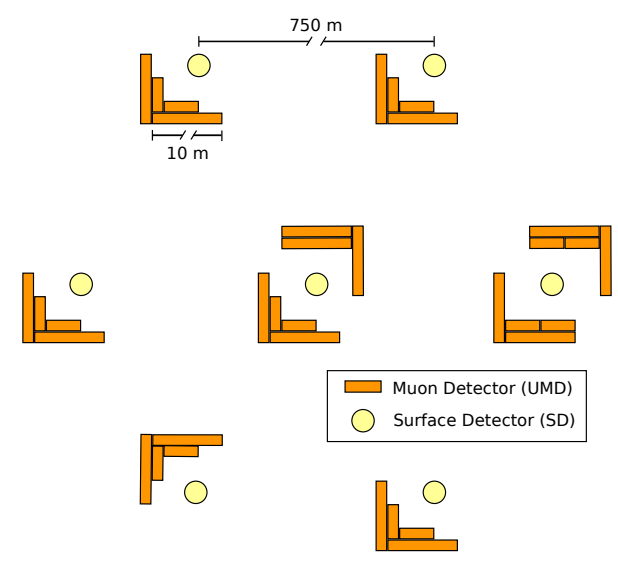

Figure 1: Layout of the AMIGA engineering array. In the prototype phase, each $30 \mathrm{~m}^{2}$ muon detector was segmented in $5 \mathrm{~m}^{2}$ and $10 \mathrm{~m}^{2}$ modules. Also, two positions were equipped with extra twin modules to assess the detector uncertainties. Two optical sensors were tested, multipixel photomultiplier tubes and silicon photomultipliers. the upgrade, surface scintillator detectors (SSDs) [4], and radio detectors [5], will be installed on top of each WCD in the SD-1500 array. These detectors aim to enhance the separation of the muonic and the electromagnetic component of air showers measured with the WCDs, more specifically, the SSDs for not very inclined showers and the radio detector for horizontal $\left(>75^{\circ}\right)$. Each of these instruments will provide complementary information on the air showers composition, which will contribute to an improved estimation of the properties of cosmic-ray primaries. In particular, the UMD will improve the cosmic ray mass identification in the ankle region of the energy spectrum. Furthermore, since it directly measures muons of a sub-sample of showers observed by the upgraded Auger surface detector, the UMD will, in particular, serve as verification and fine-tuning of the methods used to extract shower muon content using SSDs and WCDs. 


\section{The AMIGA underground muon detector}

To attain a broad dynamic range, the readout electronics of the UMD modules implements both counter and integrator modes. Schematics of the electronics can be found in Fig. 2. The counter mode independently handles the $64 \mathrm{SiPM}$ signals through a pre-amplifier, fast-shaper, and a discriminator, built within each channel of two 32-channel Application-Specific Integrated Circuits (ASICs). The discriminator signal is sampled at $320 \mathrm{MHz}$ (3.125 ns sample time) with a Field-Programmable Gate Array (FPGA) into 64 2048-bit traces. In each trace, a "1"-bit is output if the signal is above the discriminator threshold, set at 2.5 photon-equivalents (PEs) to reject most of the SiPM dark rate [6], and a "0"-bit otherwise. In this working mode, muons can be identified as sequences of " 1 "s in the binary traces. A simulated single-muon trace is shown in the middle panel of Fig. 2.

On the other hand, with the integrator mode the 64 SiPM signals are added up analogically and the result is amplified with low- and high-gain amplifiers. The signals are then sampled at $160 \mathrm{MHz}$ (6.25 ns sample time) with two Analog-to-Digital Converters (ADCs) resulting in two waveforms of 1024 samples. In the right panel of Fig. 2, a simulated trace of a single-muon signal of the integrator mode is displayed. The number of muons can then be estimated by dividing the signal charge by the mean charge of a single muon.

Both modes work in a complementary fashion. The counter mode is limited by the detector segmentation; two muons arriving at the same strip simultaneously will be counted as a single particle. This effect limits the number of muons that can be detected at the same time. The integrator mode improves this limit and extends the detector dynamic range to measure higher muon densities, thus reaching distances closer to the shower core. Furthermore, each working mode has different biases, resolutions, and systematics, and still, they both measure simultaneously the same particles. A combined analysis is foreseen [7] and could be useful to understand these features and to reduce their impact in the detection uncertainties.
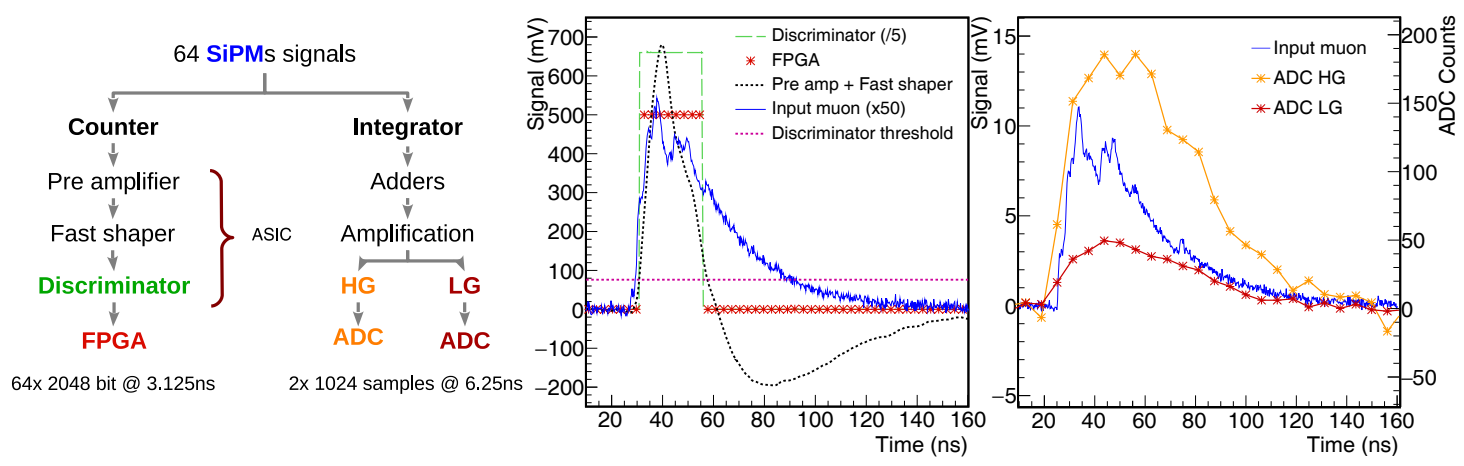

Figure 2: (Left) Schematics of the AMIGA muon detector electronics. The modules implement a counter and an integrator mode. In the counter mode, the output consists of 64 binary traces in which muons can be identified as sequences of "1". The integrator mode outputs two waveforms from which the muon number can be estimated dividing the signal charge by the mean charge of a single muon. (Middle) A simulated single-muon trace in the counter mode. The signal from the SiPM and the discriminator pulse are re-scaled for illustration. The FPGA outputs a "1"-bit in the binary trace when the fast-shaper output is above the discriminator threshold. (Right) A simulated single-muon trace in the integrator mode. The two ADC outputs (low- and high-gains) are shown. 
The main goal of the UMD is to measure the muons of extensive air showers, including their arrival time. As described before, particle counting is performed individually muon-by-muon with the counter mode and proportionally to the signal charge with the integrator mode. The read-out electronics of the counter mode produces binary signals relying on the signal amplitude while for the integrator mode the output signal is based on the total-signal charge. As SiPMs are located at an extreme of each scintillator strip, it is important to characterize the attenuation of the signal along the optical fibers and its impact on the detector efficiency. In the following sections, the optical-fiber attenuation is quantified with laboratory measurements. For this purpose, a dedicated setup was mounted on a test bench and single-muon signals were acquired with the standard UMD electronics. The event trigger was performed by a movable muon telescope and the whole length of the strips was swept in steps of $0.5 \mathrm{~m}$.

\section{The counter mode muon counting and efficiency}

To obtain the number of particles reaching the UMD with the counter mode, a counting strategy that optimizes the detector performance needs to be determined. The number of " 1 "s in the binary trace to identify muons must be selected according to the time span of the muon signal and noise, as well as an inhibition window. The inhibition window consists of a time window in which the searching process for muon identification is stopped after a muon is found. This window is optimized to neither count the same muon twice (over-counting due to a short window) nor to count two muons as one (under-counting due to a long window), and it is the same for all the UMD modules. In Fig. 3 top panel, the signal width at different positions on the scintillator strip is shown, both with laboratory and simulated data. The light-output obtained when a muon impinges the scintillator strip is attenuated by the optical fiber (see Fig. 4). Therefore, the signal width in the muon detector depends on where on the scintillator strip the muon arrived, as it can be seen in Fig. 3 top. If we consider all the signal widths within a $3 \sigma$ deviation from the mean, most of muon signals last between 12 (37.5 ns) and 4 (12.5 ns) samples. Furthermore, the

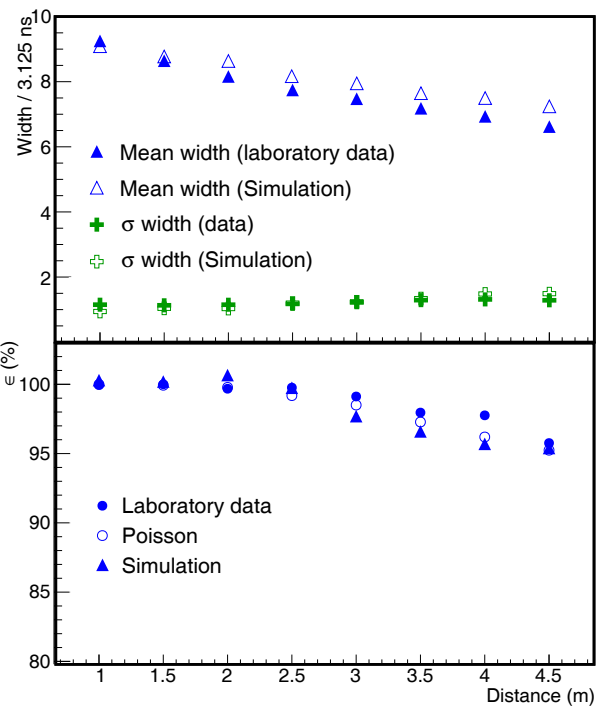

Figure 3: (Top) Width (mean) and width (RMS) over sample time in the counter mode as a function of the distance to the SiPM within the detector with both laboratory and simulated data of single muons. (Bottom) Efficiency as a function of the position estimated from laboratory data, simulations, and a Poisson prediction (see text). noise produced by dark rate of the SiPM, which can reach the 2.5 PEs discriminator threshold owing to the inner-cells crosstalk [8], has a typical width of less than $12.5 \mathrm{~ns}$. According to this data, an inhibition window of $37.5 \mathrm{~ns}$, and a minimum width of 12.5 ns maximize the signal-to-noise ratio in the counter mode. With this counting strategy, the probability of over-counting per event is reduced by a factor of $\sim 2.7$ with respect to a strategy in which a minimum width of $3.125 \mathrm{~ns}$ (one sample) is requested to count a muon. 
The resulting efficiency is shown in Fig. 3 bottom, as a function of the position on the scintillator strip, as measured in data and simulations. The estimation is compared to a simple prediction based on the measured mean number of PEs (see Fig. 4) as a function of the position on the strip. The prediction for the efficiency is obtained by integrating the Poissonian expectations given by that mean value, between the threshold (at 2.5 PEs) and infinity. The Poissonian prediction shows the efficiency loss produced by muon signals with less than 2.5 PEs, which is the counter mode threshold. Therefore, the agreement between data and prediction denotes that there is not a significant efficiency loss in the signal processing or in the signal analysis. There is also not significant over-counting due to noise and wide muon signals, which would be seen as efficiencies above $100 \%$ (a very small over-counting is then seen for the simulated data at $2 \mathrm{~m}$, resulting in $\varepsilon \approx 100.5 \%$ ). By averaging over the distance we obtained a total efficiency of $99.7 \%$ and $98.5 \%$ for the $5 \mathrm{~m}^{2}$ and $10 \mathrm{~m}^{2}$ modules, respectively.

\section{The integrator mode attenuation and calibration}

As it was mentioned before, the signal attenuation in the optical fibers has an impact on the muon identification efficiency. In the integrator mode, the number of muons is estimated by dividing the total signal charge by the mean charge of a single muon. For this reason, the signal attenuation in the optical fiber has an impact on the detector fluctuations. To understand these fluctuations, measurements of analog SiPM pulses (both muons and dark-rate PEs) were acquired with an oscilloscope at different positions on the scintillator strip. With these measurements, the number of PEs per muon was estimated in charge and amplitude as the ratio between the muon signal and a single PE. The results are shown in Fig. 4 left panel. Due to the time distribution of photons reaching the optical sensor, the curves in charge and amplitude differ significantly from each other (by a factor $\approx 2$ ). Note that the counter mode is sensitive to the signal amplitude (as it implements an amplitude threshold) whereas the integrator mode is sensitive to the signal charge. In the right panel of Fig. 4 the signal mean charge of single muons in the low- and high-gain ADC channels as a function of the position on the scintillator strip are shown. The attenuation is displayed both for laboratory and simulated data. It is apparent how the PEs attenuation has an important impact on the detector resolution since the total charge of single-muon signals between the beginning and end of the strip differs by a factor of two. Still, this impact significantly diminishes with the arrival of several muons in the whole of the detector module, for which the integrator mode is optimized to operate.

To calibrate the ADC channels, the mean charge per muon is needed. A method to obtain this value is to compare the charge in the ADC channels to the number of muons in the counter mode using shower events. In Fig. 5 left panel, the signal charge in the integrator mode is plotted against the number of muons $\left(\mathrm{N}_{\mu}\right)$ estimated with the counter mode for a $10 \mathrm{~m}^{2}$ module deployed in the Observatory. The mean charge for each $\mathrm{N}_{\mu}$ is shown along with the individual events, from which the signal dispersion can be seen. The ADC calibration is achieved by fitting the data with a linear fit, where the slope corresponds to the single-muon mean charge.

As illustration, in Fig. 5 right panel, the muon densities measured with the counter and integrator modes for an example event with a reconstructed energy of $(9.5 \pm 0.4 \pm 0.2) \times 10^{17} \mathrm{eV}$ and zenith angle of $(21.8 \pm 0.4)^{\circ}$ is displayed. The geometry and energy were obtained from the 

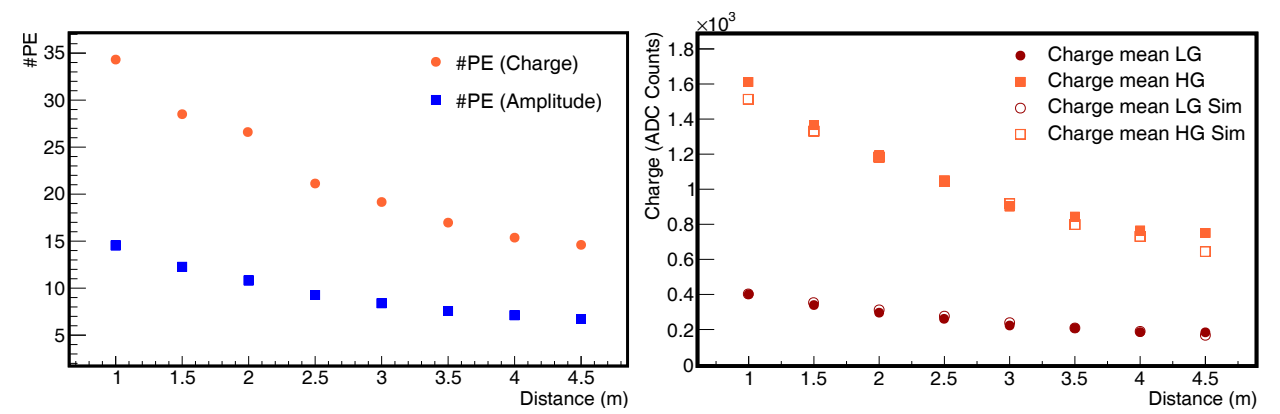

Figure 4: (Left) Number of PEs at the SiPM per muon in charge and amplitude as a function of the position on the scintillator strip. It can be seen how the curves in charge and amplitude differs since the detected photons are time distributed according to the scintillator and optical fiber decays. (Right) Mean charge as a function of the position on the scintillator strip for single-muon signals.

SD-750 reconstruction [10]. The muon lateral distribution function (MLDF) [9] was fitted using only the counter mode. Note that the reconstruction of data with the two modes is not expected to entirely agree at this point, as the final corrections for different sources of bias (from clipping corners, noise, etc. [11]) are not yet implemented. Still, it is apparent that both muon-density estimations are quite consistent for this example event.
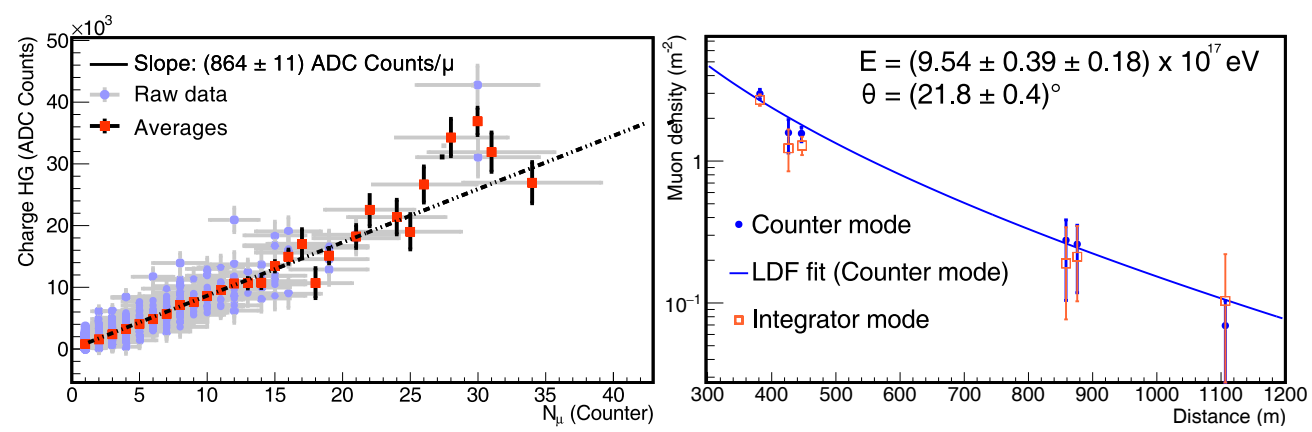

Figure 5: (Left) Signal charge in the integrator mode as a function of the number of muons estimated with the counter mode for an example UMD module. (Right) Muon lateral distribution function reconstructed with the counter mode alone for an example event. The muon densities estimated with the ADC channel are also shown for reference.

\section{Timing studies}

Before entering into the production phase and selecting SiPMs as optical sensors, multi-anode photomultipliers (PMTs) were tested in the engineering array. During this phase, over one year of calibrated PMT data (October 2015 - October 2016) was acquired [11]. Originally, the AMIGA muon detector was specifically designed to provide direct measurements of muon densities [2]. Results on the muon contents above $10^{17.5} \mathrm{eV}$ have been reported using the calibrated PMT data [12]. Nevertheless, the UMD is also suitable to study the muon timing. The measured arrival-time distribution may be used to reconstruct the shower-axis direction of each event.

To reconstruct the shower axis, the arrival time of the muons in GPS coordinates is required. Since the UMD modules do not have an integrated GPS service, the associated WCD timing is exported. The trace of the UMD modules is delayed with respect to the trace of its WCD by a fixed 
offset. Each UMD module is synchronized against its associated WCD using the arrival time of the air shower particles.

The timing of the muons observed at different positions is used to determine the arrival direction of the primary particle. The zenith $(\theta)$ and azimuth angles $(\varphi)$ of the shower axis are determined by reconstructing a shower front. To this aim, it is necessary to determine a time observable in each detector. In the SD detectors, the time observable is the start time of the signals $\left(t_{0}\right)$. Since the estimation of $t_{0}$ with a low bias requires many particles in a detector, and the UMD modules frequently contain a few muons, the $t_{0}$ estimator is not a suitable time observable for the UMD. Instead, an unbiased time estimator is provided by statistics like the median arrival time $\left(t_{50}\right)$ observed in a position. The time observable $t_{50}$, its error model, and the curvature radius $(\mathrm{R})$ are the three ingredients required to reconstruct the geometry of the air showers observed by the UMD.

The shower front is modeled as a bubble expanding at the speed of light from a center $\left(\vec{r}_{0}\right)$ located one curvature radius away from the core position $\left(\vec{r}_{c}\right)$ in the direction of the axis. The arrival time of the front at the UMD module is $t\left(\vec{r}_{i}\right)=\frac{\left\|\vec{r}_{i}-\vec{r}_{c}-\vec{r}_{0}\right\|-R}{c}+t_{c}$, where $t_{c}$ is the arrival time of the core. The front model depends on the zenith and azimuth angles and on the curvature radius through the position of the shower center $\vec{r}_{0}$. The core position is fixed with the value provided by the SD reconstruction [10]. In the left panel of Fig. 6 the geometrical reconstruction of an event that triggered the whole UMD engineering array is shown. The data corresponds to the $t_{50}$ measured in each position with respect to a plane front oriented with the axis reconstructed by the UMD. To crosscheck the reconstruction results, the distribution of the angle $(\eta)$ between the axes reconstructed by the SD and UMD is shown in Fig. 6 right panel. The angle that contains $68 \%$ of the events $\left(\eta_{68 \%}\right)$ was chosen to be the estimator of the deviation between the UMD and SD axes. For events with energy above $10^{17.5} \mathrm{eV}, \eta_{68 \%}=1.8^{\circ}$.
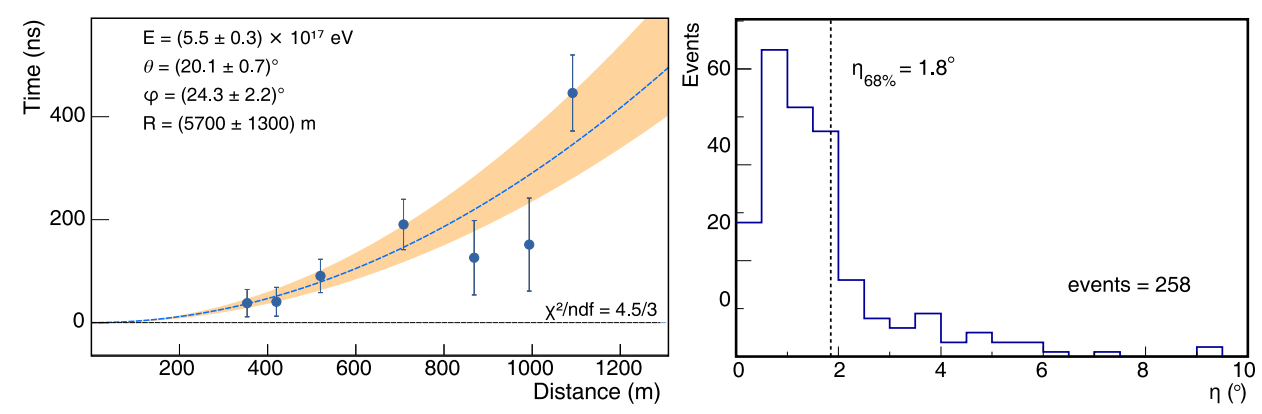

Figure 6: (Left) Delays of $t_{50}$ of an example event measured by the UMD with respect to a plane front oriented with the arrival direction reconstructed by the UMD. (Right) Angle between the axes reconstructed by the UMD and the SD for events with energy above $10^{17.5} \mathrm{eV}$.

As events with high multiplicity in the number of detectors, like the one shown in Fig 6, are rare in the engineering array ( $\sim 60 \%$ less than the total of events), the radius of curvature has to be fixed in the fit. Therefore, to estimate the curvature radius, the delay of $t_{50}$ with respect to a plane shower front oriented with the SD axis as a function of the distance was measured. For each zenith angle, the median delay $(\tilde{t})$ was computed in each distance bin (d) and fitted with a spherical front $\tilde{t}=t_{0}+\frac{d^{2}}{2 c R}$ as it can be seen in the left panel of Fig. 7. From these fits, the radius of curvature $(R)$ as a function of the zenith angle was obtained as it is displayed in the right panel of Fig 7. The radius of curvature using simulations of iron and proton primaries was also computed, using 
QGSJETII-04 as hadronic model. These results are encouraging and prove the potential of the UMD to perform studies on the time distribution of arriving muons in extensive air showers with energies above $1 \mathrm{GeV}$, which is the muon energy threshold of the UMD.
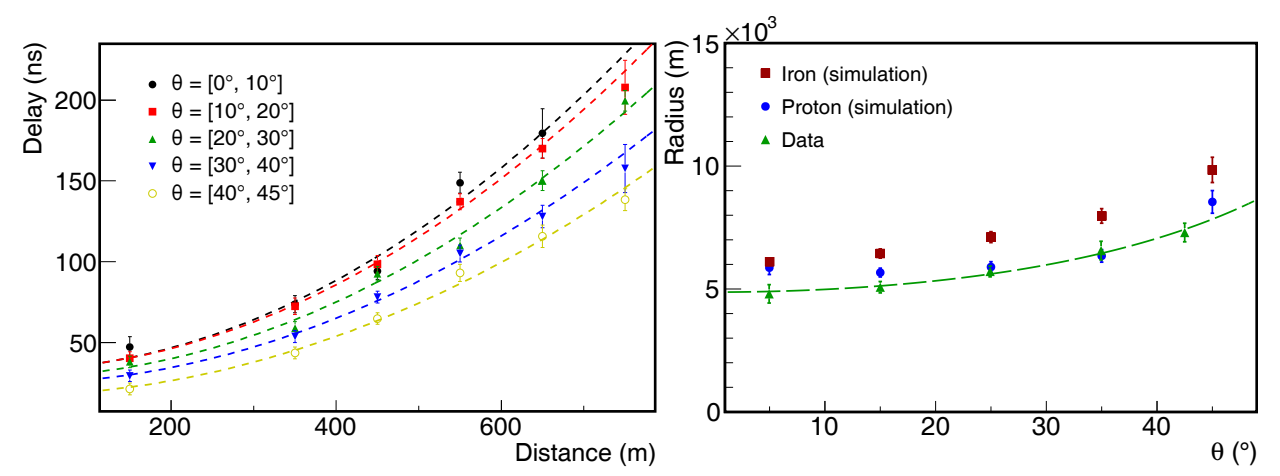

Figure 7: (Left) Residual of the UMD $t_{50}$ with respect to a plane perpendicular to the axis fitted with the SD data for each zenith angle as a function of the distance to the shower core for energies above $10^{17.5} \mathrm{eV}$. From the data, the radius of curvature is obtained. (Right) Radius of curvature as a function of the zenith angle for both data and simulations (QGSJETII-04) with proton and iron primaries.

\section{Conclusions}

The status of the AMIGA underground muon detector has been presented. The two operation modes (counter and integrator) have been described, along with their performance in the laboratory. A strategy that optimizes the signal-to-noise ratio in the counter mode has been selected based on laboratory data. Furthermore, the integrator mode has been calibrated using shower data to obtain the muon densities with both modes. In addition, a new analysis using the arrival time of muons has been presented, showing the capabilities of the UMD to reconstruct the shower geometry and to perform studies on the time distribution of the arriving muons in extensive air showers. These were conducted with modules instrumented with PMTs and will be adapted to SiPMs.

The UMD production phase - with scintillation areas of $10 \mathrm{~m}^{2}$ and SiPMs as readout - has already started. The full AMIGA array is expected to be completed by 2020 .

\section{References}

[1] The Pierre Auger Collaboration. NIM A 798 (2015) 172-213.

[2] The Pierre Auger Collaboration. JINST 11 (2016) P02012.

[3] The Pierre Auger Collaboration arXiv:1604.03637 (2016).

[4] Alvaro Taboada for the Pierre Auger Collaboration. These conference proceedings. POS (ICRC2019) 434.

[5] Marvin Gottowik for the Pierre Auger Collaboration. These conference proceedings. PoS ( ICRC2 019) 274.

[6] The Pierre Auger Collaboration. JINST 12 (2017) P03002.

[7] Darko Veberič. Appl. Opt. 51 (2012) 139-147.

[8] D. Renker, E. Lorenz. JINST 4 (2009) P04004.

[9] D. Ravignani, et al. Astropart. Phys. 82 (2016) 108-116.

[10] The Pierre Auger Collaboration. JCAP 08 (2014) 019.

[11] S. Müller for the Pierre Auger Collaboration. Ultra High Energy Cosmic Rays proceedings (2018) 02013.

[12] Federico Sánchez for the Pierre Auger Collaboration. These conference proceedings. PoS ( ICRC2 019$) 411$. 